\title{
The Analysis of Student Collaborative Work Inside Social Learning Network Analysis Based on Degree and Eigenvector Centrality
}

\author{
Andi Besse Firdausiah Mansur, Norazah Yusof, Ahmad Hoirul Basori \\ Faculty of Computing and Information Technology Rabigh, King Abdulaziz University, Kingdom of Saudi Arabia
}

\section{Article Info}

Article history:

Received Jun 10, 2016

Revised Aug 18, 2016

Accepted Aug 30, 2016

\section{Keyword:}

Authoring tools and methods Collaborative learning Cooperative learning Pedagogical issues

\begin{abstract}
Social learning network analysis is a potential approach to analyze the behaviour of students in collaborative work. However, most of the previous works focus on asynchronous discussion forum as the learning activity. Very few of them are trying to analyze the students' collaborative work while using wiki e-learning. This paper proposes the degree centrality and eigenvector method for identifying the collaborative work of students while in wiki e-learning. The log data of the Moodle e-learning system is observed that records the students' activities and actions while using wiki. The result shows that there is a close similarity between the degree centrality and the eigenvector. The result also reveals the students who obtain high outdegree values. Furthermore, Agent_1 and Agent_12 represent the students who obtained high outdegree values, which mean these two nodes are acting as source providers that able to supply information and knowledge through the network. This result also strengthened by value of closeness and betweenness where Agent_1 and Agent_12 leading on this measurement. The high closeness value of Agent_1 and Agent_12 will lead into fast spreading information since they have fastest route and has the most direct route to the other node inside the network, thus collaborative work is easy to be initialized by these Agents. This work has successfully identified collaborative work of student. This finding is believed to bring enormous benefit on the e-learning system improvement in the future.
\end{abstract}

Copyright (C) 2016 Institute of Advanced Engineering and Science. All rights reserved.

\section{Corresponding Author:}

Andi Besse Firdausiah Mansur,

Departement of Computer Science,

Faculty of Computing and Information Technology Rabigh King Abdulaziz University,

Rabigh, Makkah, Saudi Arabia.

Email: abmansur@kau.edu.sa

\section{INTRODUCTION}

Social network analysis (SNA) is one of the widely used approach to analyze the behaviour of certain community of social network. This is due to the fact that, it can represent the social relation between people [1],[2]. Teachers and researchers often employed the social network analysis (SNA) to determine the level of participants, to identify the central actors, or to recognize other structural characteristics of the social learning interactions [3].

Wiki is a collaborative tool that offers users to work together within the same system to increase the result and productivity of the teamwork [4]. Typically, it recorded the page of the original contributor of a piece of writing and those who have edited and made amendments. Several social learning activities, which are considered as collaborative work are editing, uploading, commenting and tagging [5]. The other work on Wiki is proposed by Twu in 2010. He studies the interaction among students in Chinese ESL Classroom. Their methods are divided into two parts: Attitude towards Wiki and Wiki interaction [6]. Furthermore, the 
Wikipedia system has been analyzed using centrality measurement by Korfiatis et. al [7]. They have used indegree centrality to measure how much the change that contributed by an actor that edited by another actor. They also utilized fuzzy operator to aggregate the difference between the recent version of the article and the submitted one [7]. Wiki Moodle has several features in Moodle e-learning system such as Add a new topic, Edit or Update, Comment, Link and View. It is able to give students and lecturers flexibilities to control their own Wiki.

Most of the previous research focused on analyzing the content of asynchronous discussion such as discussion forums and messaging. On the other hand, the works on Wiki still didn't touch Moodle Wiki (specifically for e-learning), they only focusing on regular Wiki such as Wikipedia. Besides that, most of the work focuses on the indegree centrality. However, they are still lacking in term of the outdegree method and other centrality methods such as closeness and betweenness. Therefore, the objective of this paper is to fill in those research gaps by focusing on implementing SNA to analyze the participation and interaction of students inside Moodle Wiki by using SNA method such as Degree Centrality, Closeness, Betweenness and Eigenvector.

Social Network Analysis (SNA) is the study of the structure of social interactions [8]. Furthermore, Social learning network analysis is the analysis of social networks in the elearning domain. Because social learning network is a new way of communication network, it can influence the teaching and learning process [9]. Previous researchers have proposed automatic document text analysis (ATA) in the student text. The text of the student's message was able to explain the emotions of the tutors and students during the process of interaction [10]-[13]. The other research analyse text messages from students categorized and analyzed according to the content of the message. This method appears to produce emotional behavior from students and tutors during the interaction. In addition, this node corresponds to a human, an agent or an actor in the community. Several techniques in SNA are the degree centrality, betweenness and closeness. These techniques can be utilized for behaviour identification. They also able to reveal behaviour of users inside social learning network [14]-[18].

The degree centrality composes of the outdegree and the indegree. The outdegree is interpreted as a number of information or knowledge that being spread from certain node (vertex) to the other node (outgoing edge). Meanwhile, the indegree is focused on calculating the number of information that a node received (ingoing edge). In addition, closeness is a technique to measure the time needed to spread the information from an initial node to another node by considering the shortest path. Betweenness is a method to determine the node that control the communication for other node inside the network (can be called as a hub). Furthermore, Eigenvector represents the connection of a node to other well connected node. This means, a node that has high Eigenvector has the potential to spread information fast and smooth inside the network. Furthermore, the other researcher proposed Computer-Aided Collaborative Decision Making (CA-CDM) that can help student to optimize their critical thinking and decision process during the study [19]. While the other try to measure the collaboration and communication of student through social network analysis. They recommend that lecturer can provide more organized events that streghthen student collaboration [20].

\section{RESEARCH METHOD}

In this research there are two main phases, first the data collection which is obtained from e-learning $\log$ data, then the second part is social network analysis for wiki. The data for analysis is the log activities of the Moodle Wiki conducted in semester 2010/2011 for the course named "Instrumentation in Analytical Chemistry" with the course code of SSC2213-01. There are several activities in Moodle Wiki such as "Add new Wiki Topic", "Edit content", "View content" and "Add Link". This activity log on Moodle Wiki is captured for three months period.

The data from Moodle Wiki is classified based on the user participation to the E-Learning system. This data is then converted into adjacency matrix in order to measure the social interaction between the users. The adjacency matrix for the data is presented in Table 1 . 
Table 1. Adjacency matrix for Wiki Data

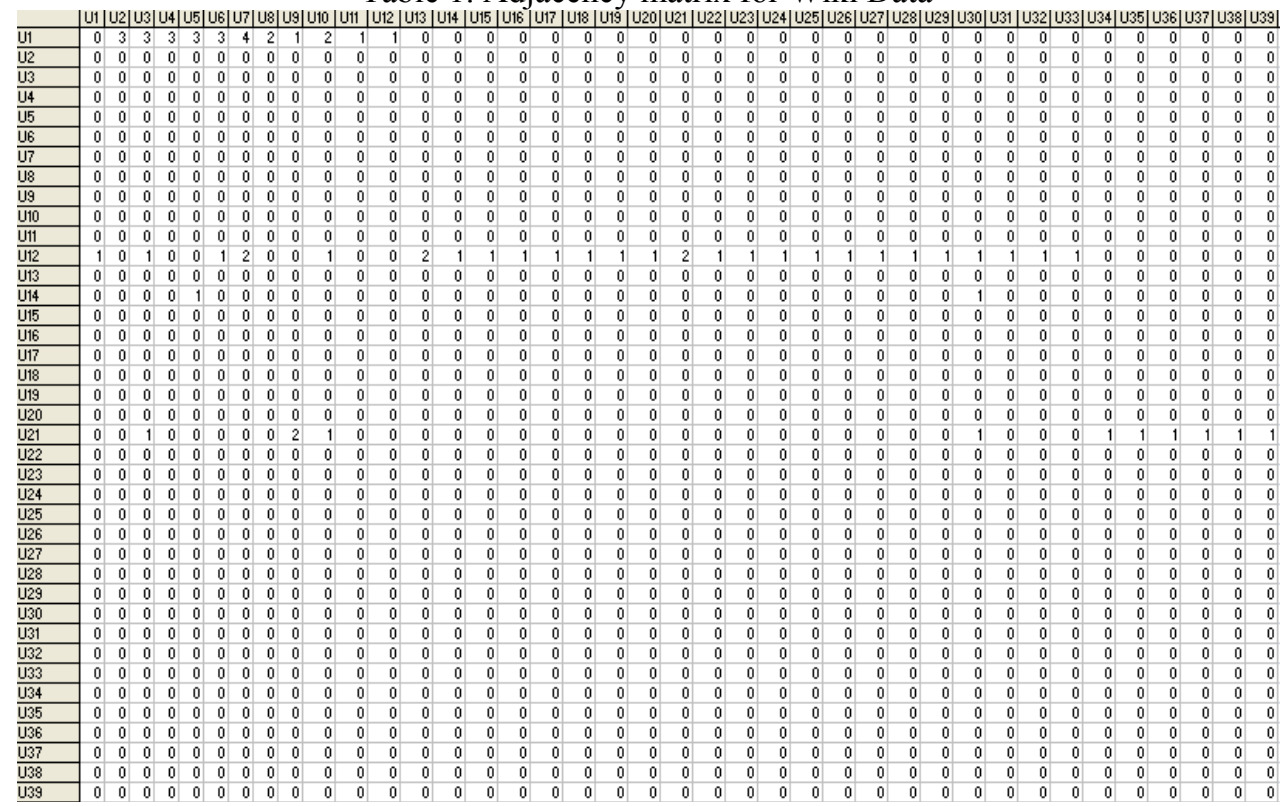

The data from the adjacency matrix is then being computed for the degree centrality, the closeness and the betweenness centrality. Table 2 shows the degree centrality which presents the indegree and outdegree results. The indegree and outdegree measurement are necessary in order to see the activity level of each student. The indegree is a calculation of the number of directed bind to the node. On the other hand, the outdegree is the number of binds that the node directs to others. In an example of a friendship relations, the indegree may be interpreted as a form of attractiveness while the outdegree is interpreted as sociability. The degree centrality of student inside social learning network can be computed through Equation 1.

For graph $\mathrm{G}:=(V, E)$ with $n$ vertices, the degree centrality $\mathrm{C}_{\mathrm{D}}(v)$ for vertex $v$ is:

$$
C_{D}(v)=\frac{\operatorname{deg}(v)}{n-1}
$$

In Table 1, the symbol $U$ is symbolized as Agent_1. It can be seen that Agent_12 (U12), Agent_1 (U1) and Agent 21 (U21) have high outdegree values. These mean that those students give significant contribution to other Wiki users in terms of editing, adding, or even updating the Wiki. On the other hand, Agent_7, Agent_6, Agent_5, Agent_3 and Agent_10 receive high indegree values. This mean that the Wiki page that being edited by these students have been edited by the most users.

Table 2. Degree Centrality measure for Wiki data

\begin{tabular}{ccc}
\hline Name & Indegree & Outdegree \\
\hline Agent_1 & 1.00 & 26.00 \\
Agent_2 & 3.00 & 0 \\
Agent_3 & 5.00 & 0 \\
Agent_4 & 3.00 & 0 \\
Agent_5 & 4.00 & 0 \\
Agent_6 & 4.00 & 0 \\
Agent_7 & 6.00 & 0 \\
Agent_8 & 2.00 & 0 \\
Agent_9 & 3.00 & 0 \\
Agent_10 & 4.00 & 0 \\
Agent_11 & 1.00 & 0 \\
Agent_12 & 1.00 & 29.00 \\
Agent_13 & 2.00 & 0 \\
Agent_14 & 1.00 & 2.00 \\
Agent_15 & 1.00 & 0 \\
Agent_16 & 1.00 & 0 \\
Agent_17 & 1.00 & 0 \\
Agent_18 & 1.00 & 0 \\
Agent_19 & 1.00 & 0 \\
& &
\end{tabular}

\begin{tabular}{ccc}
\hline Name & Indegree & Outdegree \\
\hline Agent_20 & 1.00 & 0 \\
Agent_21 & 1.00 & 11.00 \\
Agent_22 & 1.00 & 0 \\
Agent_23 & 1.00 & 0 \\
Agent_24 & 1.00 & 0 \\
Agent_25 & 1.00 & 0 \\
Agent_26 & 1.00 & 0 \\
Agent_27 & 1.00 & 0 \\
Agent_28 & 1.00 & 0 \\
Agent_29 & 1.00 & 0 \\
Agent_30 & 1.00 & 0 \\
Agent_31 & 1.00 & 0 \\
Agent_32 & 1.00 & 0 \\
Agent_33 & 1.00 & 0 \\
Agent_34 & 1.00 & 0 \\
Agent_35 & 1.00 & 0 \\
Agent_36 & 1.00 & 0 \\
Agent_37 & 1.00 & 0 \\
Agent_38 & 1.00 & 0 \\
Agent_39 & 1.00 & 0 \\
\hline
\end{tabular}


Table 3 shows the closeness and betweenness centrality. Within a graph, the betweenness is the centrality measure of a vertex. Vertices have high betweenness value when they occur on many shortest paths. For the graph $G:=(V, E)$ that has vertices $n$ then the betweenness $C_{B}(v)$ for vertex $v$ can be measured using several steps below:

1. For each pair of vertices $(s, t)$, calculate all shortest paths between them.

2. For each pair of vertices $(s, t)$, determine the fraction of shortest paths that pass through the vertex in question(here, vertex $v$ ).

3. Sum this fraction over all pairs ofvertices $(s, t)$, refer to Equation 2.

$$
C_{B}(v)=\sum_{s \neq v \neq t \in V} \frac{\sigma_{s t}(v)}{\sigma_{s t}}
$$

where $\sigma_{s t}$ is the number of shortest paths from $s$ to $t$ and $\sigma_{s t}(v)$ is the number of shortest paths from $s$ to $t$ that go by vertex $(v)$.

Furthermore, the closeness $C_{C} v$ for a vertex $v$ is the reciprocal of the sum of geodesic distances to all other vertices of V(Sabidussi, 1966). Instead of using geodesic distance to measure closeness, Noh and Rieger (2003) proposed the random-walk centrality that is a measure of the speed with which randomly walking messages reach a vertex from elsewhere in the network a sort of random-walk version of closeness centrality (Noh and Rieger, 2004). In essence, it measures the harmonic mean length of paths ending at a vertex $i$. $i$ is small when there are many short paths connecting it to other vertices (Stephenson and Zelen, 1989). he equation of closeness has been improved to overcome network vulnerability. This condition is useful and makes closeness computation for disconnected graph 3 become easy.

$$
C_{C}(v)=\sum_{t \in V \backslash v} 2^{-d_{G}(v, t)}
$$

Table 3 presents the findings of the betweenness and the closeness calculation based on the adjacency matrix in Table 1. Agent_1 (Student_1) and Agent_12 (Student_12) have the highest closeness values which mean that these students are particularly important because their distances are closest to other students in the network. In other words, the closest student distance, the fastest information will be delivered. Furthermore, betweenness also show a compelling result where Agent_12 and Agent 21 become the main actor hub who connect some nodes to other nodes. The role of actor hub is crucial for the information flow inside the network.

\begin{tabular}{|c|c|c|c|c|c|}
\hline Name & Closeness & Betwenness & Name & Closeness & Betwenness \\
\hline Agent_1 & 0.011 & 5.000 & Agent_20 & 0.000 & 0.000 \\
\hline Agent 2 & 0.00 & 0.000 & Agent 21 & 0.001 & 12.000 \\
\hline Agent 3 & 0.00 & 0.000 & Agent 22 & 0.000 & 0.000 \\
\hline Agent 4 & 0.000 & 0.000 & Agent 23 & 0.000 & 0.000 \\
\hline Agent 5 & 0.000 & 0.000 & Agent_24 & 0.000 & 0.000 \\
\hline Agent 6 & 0.000 & 0.000 & Agent 25 & 0.000 & 0.000 \\
\hline Agent 7 & 0.000 & 0.000 & Agent_26 & 0.000 & 0.000 \\
\hline Agent 8 & 0.000 & 0.000 & Agent_27 & 0.000 & 0.000 \\
\hline Agent_9 & 0.000 & 0.000 & Agent_28 & 0.000 & 0.000 \\
\hline Agent 10 & 0.000 & 0.000 & Agent 29 & 0.000 & 0.000 \\
\hline Agent 11 & 0.000 & 0.000 & Agent 30 & 0.000 & 0.000 \\
\hline Agent 12 & 0.016 & 31.000 & Agent 31 & 0.000 & 0.000 \\
\hline Agent_13 & 0.000 & 0.000 & Agent 32 & 0.000 & 0.000 \\
\hline Agent 14 & 0.001 & 1.500 & Agent 33 & 0.000 & 0.000 \\
\hline Agent_15 & 0.000 & 0.000 & Agent_34 & 0.000 & 0.000 \\
\hline Agent 16 & 0.000 & 0.000 & Agent 35 & 0.000 & 0.000 \\
\hline Agent_17 & 0.000 & 0.000 & Agent_ 36 & 0.000 & 0.000 \\
\hline Agent_18 & 0.000 & 0.000 & Agent_37 & 0.000 & 0.000 \\
\hline \multirow[t]{2}{*}{ Agent_19 } & 0.000 & 0.000 & Agent 38 & 0.000 & 0.000 \\
\hline & & & Agent 39 & 0.000 & 0.000 \\
\hline
\end{tabular}

Table 3. Closeness and Betweenness Centrality measure for Wiki data 
In addition, this section intends to reveal more the behaviour of collaborative work of student by measuring the popularity of student using eigenvector centrality. Eigenvector centrality is able to measure "How well is this person connected to other well connected people", so the highest eigenvector can be considered as the attractive person inside the network(Cheliotis, 2006). Symmetrize is a method to change "directed" or "asymmetric" network data into "undirected" or "symmetric" data. There are various methods to symmetrize data. In this study, all symmetrize data are to cover the evaluation of collaborative work inside Moodle Wiki. The data is obtained from the Wiki interaction presented in Table 1. The measurement of eigenvector centrality can be referred to the Equation 4.

$$
\begin{aligned}
& \lambda v=A v \\
& (A-\lambda I) v=0
\end{aligned}
$$

where $\mathrm{A}$ is adjacency matrix from graph,

$\lambda$ is eigen value and $v$ is eigen vector.

The symmetrize method is described as follow:

\section{- Maximum}

If there are two actors $\mathrm{A}$ and $\mathrm{B}$, then the strongest tie among them is chosen to be representative of a tie for A and B. The data of wiki that has been adjusted into matrix adjacency on Tablel is analyzed through Eigenvector centrality using symmetrize maximum. The distribution of Eigenvector centrality scores is presented in Table 4 with mean 0.102 .

Table 4. Distribution score for Eigenvector centrality-MAX

\begin{tabular}{cl}
\hline Measures & Value \\
\hline Mean & 0.102 \\
Std.Dev. & 0.123 \\
Min. & 0.017 \\
Max. & 0.624 \\
\hline
\end{tabular}

The relationship between Agent through Eigenvector centrality was illustrated clearly through Concentric Diagram as shown in Figure 1. Agent_1 becomes centre of other Agent. This means that Agent 1 can be considered as well-connected people in the real world. This kind of person is particularly essential to make sure that the collaborative work become a success or not.

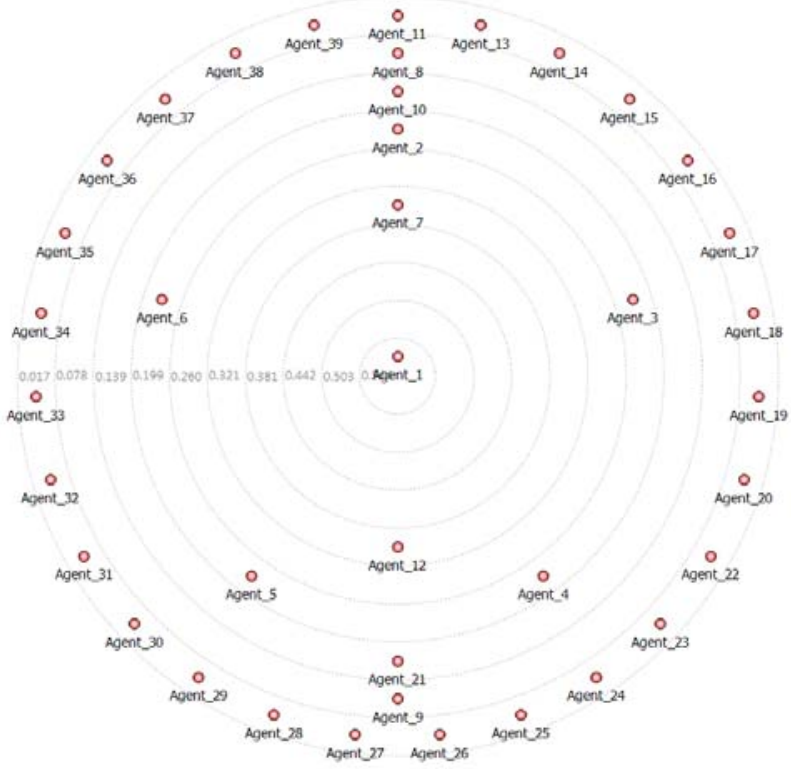

Figure 1. Concentric diagram for Eigenvector centrality with Maximum Symmetrize 
- Minimum

Exemplifies the power of the symmetric tie among A and B as being the feeble of the ties AB or BA or generally is known as weakest link. The second evaluation of the collaborative work inside Moodle wiki is through Eigenvector with Minimum Symmetrize. The distribution of Eigenvector centrality score is described in Table 5 with mean 0.036 .

Table 5. Score for Eigenvector centrality vector with Minimum Symmetrize

\begin{tabular}{cc}
\hline Measures & Value \\
\hline Mean & 0.036 \\
Std.Dev. & 0.156 \\
Min. & 0 \\
Max. & 0.814 \\
\hline
\end{tabular}

This Eigenvector concentric is described clearly in Figure 2. In this figure, Agent_12 becomes the center of the others agent and acts as the most well connected people.

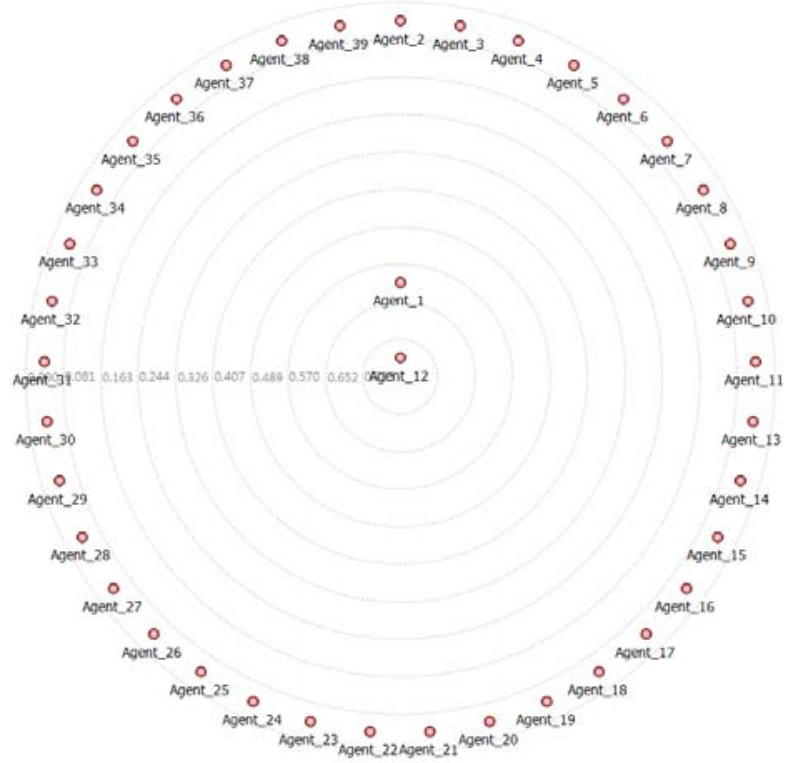

Figure 2. Concentric diagram for Eigenvector centrality with Minimum Symmetrize

- Average

Demonstrates the power of the symmetric tie among $\mathrm{A}$ and $\mathrm{B}$ as the plain average of the ties $\mathrm{AB}$ and BA. The third evaluation of the collaborative work inside Moodle wiki is through Eigenvector with Average Symmetrize. The distribution of Eigenvector centrality score is described in Table 6 with mean 0.103 .

Table 6. Score for Eigenvector centrality vector with Average Symmetrize

\begin{tabular}{cc}
\hline Measures & Value \\
\hline Mean & 0.103 \\
Std.Dev. & 0.123 \\
Min. & 0.016 \\
Max. & 0.617 \\
\hline
\end{tabular}

Figure 3 presents the positions of the agent among other agents. Agent_1 is located at the centre of the concentric diagram, and Agent_3 is placed in the third layer. Meanwhile, Agent_ 7 is positioned in the fourth layer. 


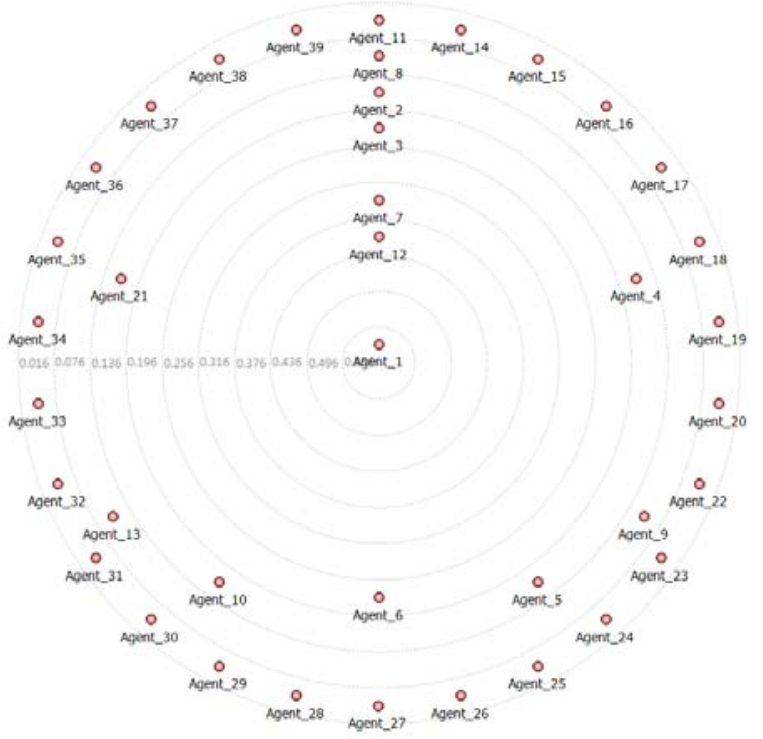

Figure 3. Concentric diagram of the Eigenvector centrality with Average Symmetrize

- Lower Half

Utilizes one half values of the matrix from the other half. If the "senders" become the focus of the network properties, then it will set the lower half equals to the upper half. However, if the focus of the network has changed to "receiver", then the upper half equals to the lower half. The fourth symmetrize of Eigenvector centrality is the Lower half. Table 7 displays the measurement values for the Eigenvector distribution and Figure 4 illustrates the agent behaviour inside the concentric diagram.

Table 7. Score for Eigenvector centrality with Lower half Symmetrize

\begin{tabular}{cc}
\hline Measures & Value \\
\hline Mean & 0.067 \\
Std.Dev. & 0.145 \\
Min. & 0 \\
Max. & 0.623 \\
\hline
\end{tabular}

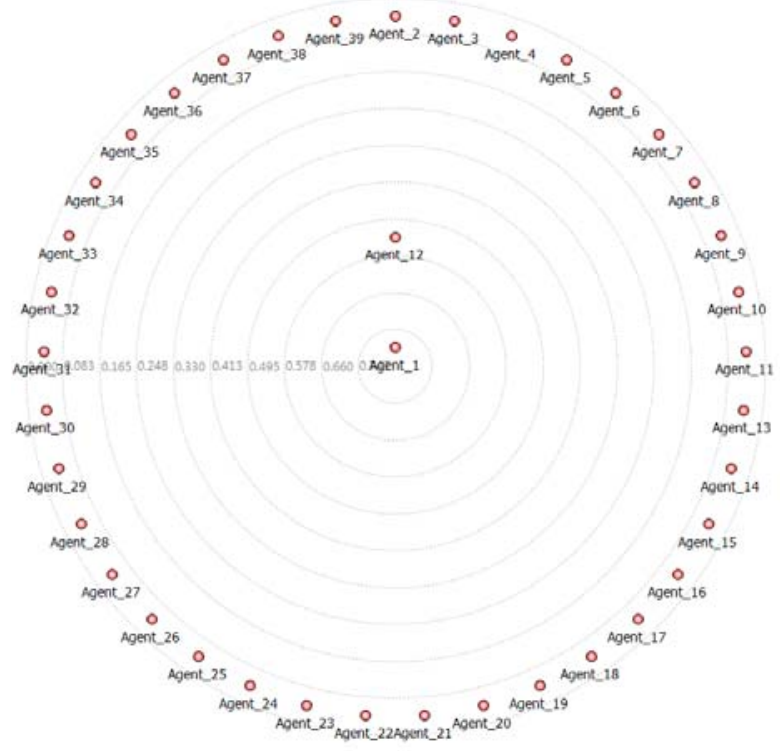

Figure 4. Concentric diagram for Eigenvector centrality with Lower half Symmetrize 
- Upper Half

Evaluates the values in unit $\mathrm{AB}$ and $\mathrm{BA}$, and returns a value based on the test function. For example, if Upper > Lower and $\mathrm{AB}=2, \mathrm{BA}=6$, then the application would select value "6," since upper value $(\mathrm{AB})$ was not larger than the lower value (BA). The last symmetrize that will be used in this evaluation is upper half. The result of Eigenvector using this mode is presented in Table 8. The details of the interaction among agents are portrayed in Figure 5 and 6.

Table 8. Score for Eigenvector centrality with Upper half Symmetrize

\begin{tabular}{cc}
\hline Measures & Value \\
\hline Mean & 0.085 \\
Std.Dev. & 0.136 \\
Min. & 0.004 \\
Max. & 0.701 \\
\hline
\end{tabular}

Figure 5 portrays the layered diagram in which Agent_1 is at the centre, followed by Agent 7 , Agent4, Agent_3, Agent 5, Agent_6 and so on. The position of the agent is determined according to the Eigenvector value.

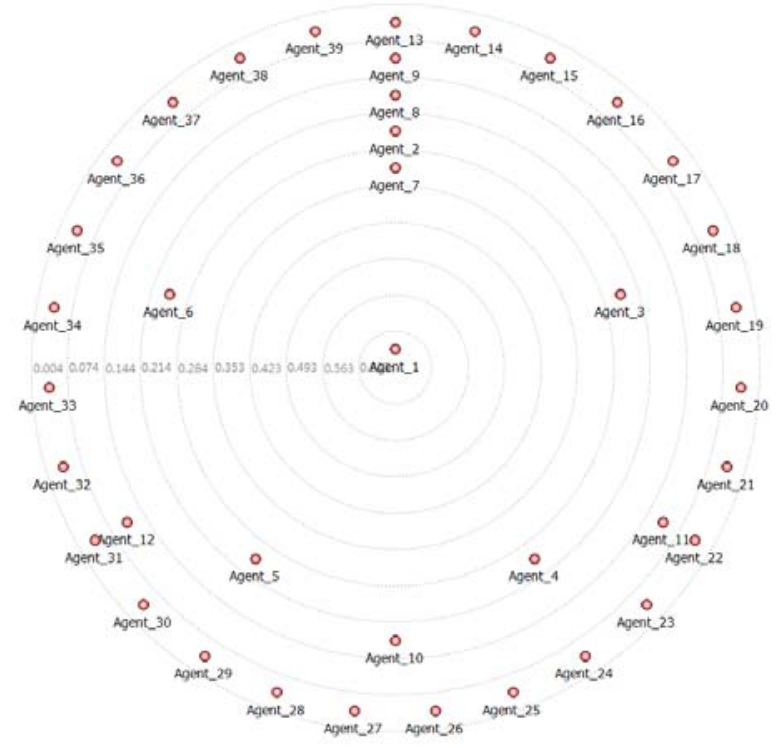

Figure 5. Concentric diagram for Eigenvector centrality with Upper half Symmetrize 


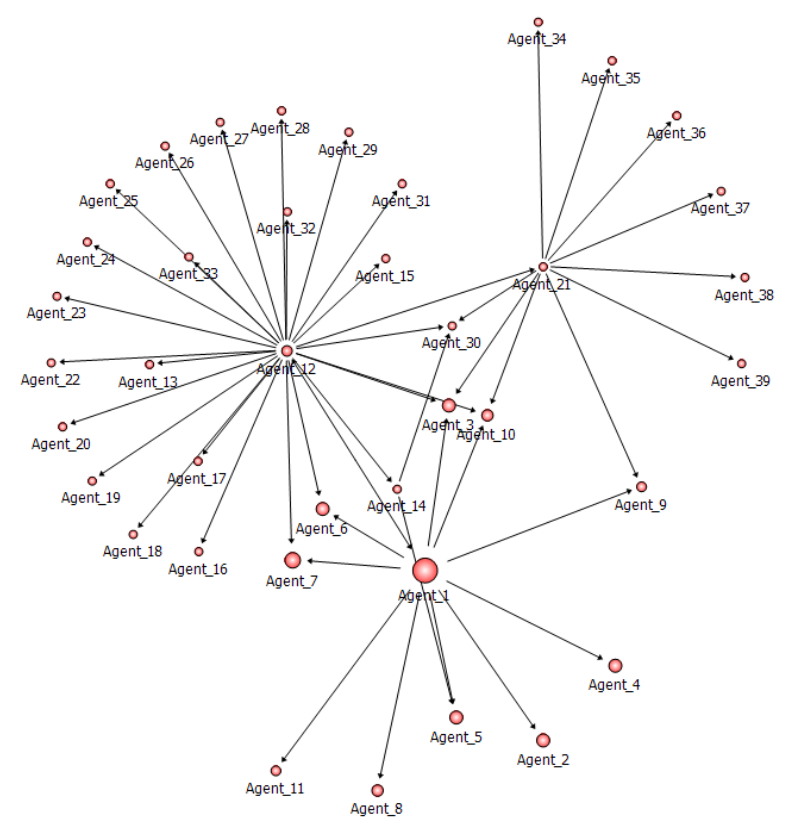

Figure 6. Spring diagram for Eigenvector centrality with Upper half Symmetrize

Figure 6 shows the spring model of Eigenvector value through Upper symmetrize. From Figure 6, Agent_1 is seen as the most well connected node that able to maintain relationships with Agent 12 and Agent_21. Moreover, the second biggest Eigenvector value in this symmetrize mode is Agent 7 . This is due to the fact that it ables to connect with Agent_1 and Agent_12.

\section{RESULTS AND CONCLUSION}

This section summarizes the analyzes result of the evaluations conducted on the collaborative work inside Moodle wiki. The measurement approaches being considered are the Degree Centrality, Betweenness, Closeness, and Eigenvector centrality. The result and analysis of these approaches are presented in Table 9. In this table, the symbol "Agent" is used to represent the student's name. The data for the Degree centrality, Closeness, and Betweenness are obtained from Table 1 and 2.

Table 9. The result and analysis of the approaches used to evaluate the collaborative work in Wiki Moodle

\begin{tabular}{|c|c|c|c|}
\hline Eigenvector Centrality & Degree Centrality & Closeness & Betweenness \\
\hline $\begin{array}{l}\text { In the Eigenvector centrality, } \\
\text { Agent_1 and Agent_12 are } \\
\text { recognized as the most well } \\
\text { connected to other well } \\
\text { connected node inside network }\end{array}$ & $\begin{array}{l}\text { Agent_12 leading the } \\
\text { outdegree values by } 29 \text { and } \\
\text { followed by Agent_1 and } \\
\text { Agent_21. }\end{array}$ & $\begin{array}{l}\text { Agent_12 obtains the highest } \\
\text { score for closeness with } \\
0.016 \text { and then pursued by } \\
\text { Agent_1. }\end{array}$ & $\begin{array}{l}\text { Agent_12 receives the highest } \\
\text { score by } 31 \text {. Next is Agent_21 } \\
\text { with the score } 15 \text { and finally, } \\
\text { Agent_1 with the score } 5\end{array}$ \\
\hline
\end{tabular}

Table 9 illustrates the results from different SNA method to analyze the interaction inside Wiki Moodle. From overall observation, Agent_1 and Agent_12 have played a vital role for the network. Based on their Eigenvector value, these two agents are the most well connected node. This means that these two students are popular and are able to manage the collaborative work. Furthermore, Agent_1 and Agent_12 also obtain high outdegree value, which mean that these two students are acting as the source provider who supply information and knowledge through the network. These results also strengthened by the value of closeness and betweenness where Agent_1 and Agent_12 also leading on this measurement. The high closeness value of Agent_1 and Agent_12 lead into fast spreading information since they have fastest route and have the most direct route to the other node inside the network. The analysis result of the Eigenvector centrality has relations with the social learning network analysis for identifying collaborative work inside Moodle Wiki. According to Cheliotis, G. (2010), "How well is this person connected to other well connected people?" is the fundamental of Eigenvector centrality measurement. With this principle, the analysis result 
also recognized that Agent_1 and Agent_12 become the key point on information distribution since they are the most well connected node. Finally, the results from the Degree centrality, Closeness, Betweenness, Hierarchical and MST clustering also support the principle, in which we can claim that Agent_1 and Agent_12 are the central nodes inside the network that hold a vital role for information circulation among the nodes.

\section{ACKNOWLEDGEMENTS}

Authors are grateful to Faculty of Computing and Information Technology Rabigh, King Abdulaziz University, Kingdom of Saudi Arabia

\section{REFERENCES}

[1] J. Ji, et al., "Maximizing the Community Coverage of Influence through a Social Network," AISS: Advances in Information Sciences and Service Sciences, vol/issue: 3(9), pp. 339 346, 2011. http://dx.doi.org/10.4156/aiss.vol3.issue9.44.

[2] B. Zhou and C. Wu, "Semantic Model for Social Networking Federation," Aiss: Advances in Information Sciences and Service Sciences," vol/issue: 3(11), pp. 213 223, 2011.

[3] Erlin B., et al., "Analyzing Online Asynchronous Discussion Using Content and Social Network Analysis," in IEEE Ninth International Conference On Intelligent Systems Design And Applications, 2009. http://dx.doi.org/10.1109/isda.2009.40.

[4] F. Ortiza and R. Fraile, "Social Network Featuring Entertainment, Culture And Technology," in Spanish Universities: The Infocampus Project The Open Information Systems Journal, vol. 3, pp. 48-53, 2009. http://dx.doi.org/10.2174/1874133900903010048.

[5] S. J. Kepp and H. Schorr, "Analyzing Collaborative Learning Activities in Wikis Using Social Network Analysis," in Chi, Spotlight on Work in Progress Session 2, April 4-9, Boston, Ma, Usa. ACM, 2009.

[6] H. L. Twu, "A Predictive Study of Wiki Interaction: Can Attitude toward Wiki Predict Wiki Interaction in HighContext Cultures Groups?” Journal of Educational Technology Development and Exchange, vol/issue: 3(1), pp. 5768, 2010.

[7] N. T. Korfiatis, et al., "Evaluating Authoritative Sources Using Social Networks: An Insight from Wikipedia," Online Information Review, vol/issue: 30(3), pp. 252 - 262, 2006.

[8] S. Wasserman and K. Faust, "Social Network Analysis," Cambridge University Press, 1994. http://dx.doi.org/10.1017/CBO9780511815478.

[9] M. F. Delaat, et al., "Online teaching in networked learning communities: A multi-method approach to studying the role of the teacher," International Journal of Web-Based Communities, 2006. Doi:10.1007/s11251-006-9007-0.

[10] C. Spadavecchia and C. Giovannella, "Monitoring Learning Experiences and Styles: The Socio-Emotional Level," in 10th IEEE International Conference on Advanced Learning Technologies, 2010. http://dx.doi.org/10.1109/icalt.2010.129.

[11] G. Cheliotis, "Social Network Analysis (SNA): Including a Tutorial on Concepts and Methods," Communications and New Media, National University of Singapore, 2006. [Accessed 27 January 2013].

[12] P. Drazdilova, et al., "Analysis of Relations in E-Learning," in IEEE/WIC/ACM International Conference on Web Intelligence and Intelligent Agent Technology, 2008. Doi: 10.1109/WIIAT.2008.250.

[13] Erlin B., et al., "Integrating Content Analysis and Social Network Analysis for Analyzing Asynchronous Discussion Forum," in IEEE, 2008. http://dx.doi.org/10.1109/itsim.2008.4631996.

[14] I. Hamulic and N. Bijedic, "Social Network Analysis in Virtual Learning Community at Faculty of Information Technologies (Fit), Mostar," Elsevier-Procedia Social and Behavioral Sciences, vol. 1, pp. 2269-2273, 2009. http://dx.doi.org/10.1016/j.sbspro.2009.01.399.

[15] A. Martinez, et al., "Combining Qualitative Evaluation And Social Network Analysis For The Study Of Classroom Social Interactions," Computer And Education, Elsevier, vol. 41, pp. 353-368, 2003. http://dx.doi.org/10.1016/j.compedu.2003.06.001.

[16] J. D. Noh and H. Rieger, Phys. Rev. Lett., pp. 92, 2004.

[17] G. Sabidussi, "The Centrality Index of a Graph,” Psychometrika, vol. 31, pp. 581-603, 1966. http://dx.doi.org/10.1007/BF02289527.

[18] K. A. Stephenson and M. Zelen, "Rethinking Centrality: Methods and Examples," Social Networks, vol. 11, 1989. http://dx.doi.org/10.1016/0378-8733(89)90016-6.

[19] U. Asgher, et al., "Analysis and Modeling of Academia's Collaborative Decision Support System Based on Key Performance Indicators and Degree of Certainty," the 6th International Conference on Applied Human Factors and Ergonomics (AHFE 2015) and the Affiliated Conferences, AHFE, vol. 3, pp. 4084-4089, 2015.

[20] G. Han, et al., "Using Social Network Analysis to Measure Student Collaboration in an Undergraduate Capstone Course," North American Colleges and Teachers of Agriculture (NACTA), vol/issue: 60(2), 2016. 


\section{BIOGRAPHIES OF AUTHORS}
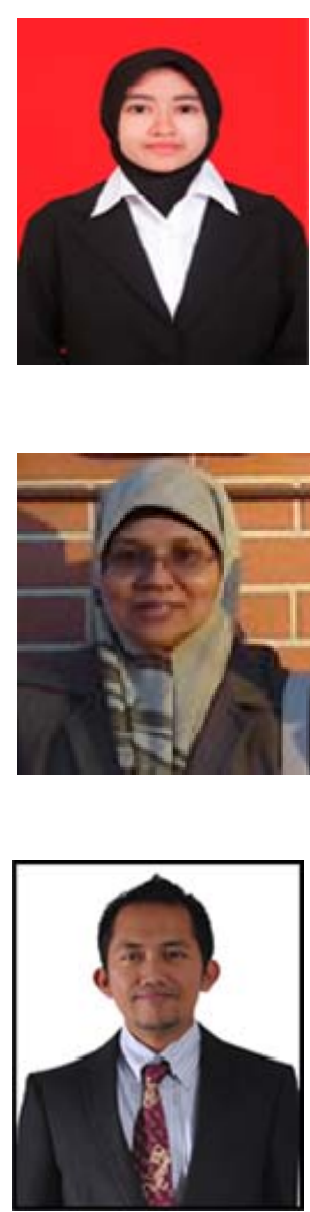

Andi Besse Firdausiah Mansur, received B.Sc (Mathematics), in 2004 and MSc(Software Engineering) in 2009. Furthermore, she obtained Ph.D. degree in Software Engineering from Universiti Teknologi Malaysia, Johor Bahru, Johor, in 2013. From 2013 to present, she is assistant professor at Faculty of Computing and Information Technology Rabigh, King Abdulaziz University. She is member of reviewer board in some international journal. Her research interest: Social Network Analysis, E-learning, Clustering, Data Mining and Mathematics.

Norazah Yusof, received PhD (System and Science Management), in 2005. She is Associate Professor at Faculty of Computing and Information Technology Rabigh, King Abdulaziz University. Her research interest includes Social Network Analysis, E-learning system, Soft Computing (Fuzzy Inference System, Neural Network, Rough Set, Genetic Algorithm) and Software Engineering.

Ahmad Hoirul Basori, was born in Wirowongso Village, jember City, Indonesia, in 1982. He received the B.Sc (Software Engineering) from Institut Teknologi Sepuluh Nopember Surabaya in 2004 and the Ph.D (Computer Graphics) from Universiti Teknologi Malaysia, Johor Bahru, Johor, in 2011. From 2004 to present, he was a lecturer with the Department of Informatics, Faculty of Information Teknologi, Institut Teknologi Sepuluh Nopember Surabaya, Indonesia. In 2011, he has appointed as Assistant Professor with the Department of Computer Graphics and Multimedia, Universiti Teknologi Malaysia. Currently, he is appointed as Associate Professor of Faculty of Computing and Information Technology Rabigh, King Abdulaziz University. He is the member of Editorial board of some international journal, more than 32 articles, and also a member of professional membership IEEE, ACM SIGGRAPH, IAENG and Senior Member of IACSIT. His research interests include Computer Graphics, Facial Animation, Cloth Simulation, Medical Visualization, Haptic Interaction, Man Machine Interaction and Robotics. 